\title{
Detection of Skin Diseases from Dermoscopy Image Using the combination of Convolutional Neural Network and One-versus-All
}

\author{
Kemal Polat $^{1 *}$, Kaan Onur Koc ${ }^{2}$ \\ ${ }^{1}$ Department of Electrical and Electronics Engineering, Faculty of Engineering, Bolu Abant Izzet Baysal University, \\ 14280, Bolu, Turkey, Email: kpolat@ibu.edu.tr \\ ${ }^{2}$ Graduate School of Natural Sciences, Department of Electrical and Electronics Engineering, Bolu Abant Izzet Baysal \\ University, 14280, Bolu, Turkey, Email: kaanonurkoc@gmail.com \\ ${ }^{*}$ Corresponding Author
}

How to cite this paper: Kemal Polat, Kaan Onur Koc (2020). Detection of Skin Diseases from Dermoscopy Image Using the combination of Convolutional Neural Network and One-versus-All. Journal of Artificial Intelligence and Systems, 2, 80-97. https://doi.org/10.33969/AIS.2020.21006.

Received: January 25, 2020

Accepted: February 5, 2020

Published: February 10, 2020

Copyright (C) 2020 by the author(s) and Institute of Electronics and Computer. This work is licensed under the Creative Commons Attribution International License (CC BY 4.0).

http://creativecommons.org/licenses/by/4.0/

\section{Open Access}

\begin{abstract}
The use of deep learning in the field of image processing is increasing. In this study, a new method based on Convolutional Neural Network is proposed to detect skin diseases automatically from Dermoscopy images. Skin cancer is one of the common diseases in the community. In this article, the skin images were taken from the data HAM10000 dataset prepared by Philipp Tschandl. There are seven classes in the skin disease data set; Actinic keratoses and intraepithelial carcinoma, Basal cell carcinoma, Benign keratosis, Dermatofibroma, Melanoma, Melanocytic type and Vascular lesions. To classify the skin diseases automatically, two different methods have been proposed: i) Alone Convolutional Neural Network model, and ii) the combination of Convolutional Neural Network and one- versus- all. In the proposed method, we have not used any pre-processing method to classify them. The raw dermatology images taken from the dataset have been given to the input of Convolutional Neural Network and then trained and tested by these images. In the second proposed method, seven different models having two-classes have been composed and then combined with the one-versus-all approach. While alone, Convolutional Neural Network obtained 77\% classification accuracy in the detection of skin disease with seven classes, the combination of Convolutional Neural Network and one-versus-all approach achieved 92.90\% accuracy. The obtained results have shown that the proposed method is very promising in the classification of skin disease from Dermoscopy images.
\end{abstract}

\section{Keywords}

Deep Learning, Skin Cancer, Convolutional Neural Network, Artificial Neural Networks, Image Processing.

\section{Introduction}

In this paper, skin disease classification has been done using two different methods including the alone Convolutional Neural Network and the combination of CNN and one-versus-all (OVA). 
Malignant melanoma occurring in skin tissue and to death is a type of skin cancer that can cause [1, 2]. With early diagnosis, the disease can be cured. In the detection of medical diseases, Computer-Aided Diagnosis (Computer) Aided Diagnosis CAD) systems for the detection of skin cancer can also help patients and physicians [2]. Dermoscopy is a non-invasive skin imaging technique. Malignant melanoma is one of the fastest-growing cancers in the world. Early diagnosis is especially important because if diagnosed early, melanoma can be treated with a simple excision [3].

In this study, we have used the convolutional neural network (CNN) as the classifier method. Convolutional neural network (CNN) is one of the best among deep artificial intelligence methods used in image processing. CNN takes an input image to train its network and produces an output that matches the input image using the existing image dataset. A neural network draws a path based on similarity connections of biological nerves interconnected with each other and thus creates a learning structure. The performance of CNN also varies depending on how many input images there are and how many convolutions are used [4]. Deep learning methods can be seen as a useful tool for dermatologists to detect lesions better. A CNN model that works to obtain a diagnostic result from an image is similar to the training and learning process of a dermatologist [4].

In the literature, there are many works regarding skin disease diagnosis using deep learning and machine learning techniques. Suhail M.Odeh et al. used four different models including k-NN (k-Nearest Neighbor), the combination of k-NN and genetic algorithm, Artificial Neural Networks with Genetic Algorithm, and Adaptive Neuro-Fuzzy Inference System to diagnose the skin cancer from the lesion images [5]. In the work of Fekrache Dalila et al. [6], they proposed a hybrid approach to diagnosing melanoma and benign skin lesions. In their method, to segment, the images, ant colony optimization method has been used to extract the features from these lesions and then used the K-Nearest Neighbor (KNN) and Artificial Neural Network (ANN) to classify the melanoma and benign skin lesions [6]. Wiem Abbes et al. proposed an ontology-based classification model for the classification of skin lesions. In their method, they used the Bag-of-Words (BoW) modeling to extract the features from the images. Then, the support vector machine (SVM) has been used to classify the skin lesions [7]. Pedro Pedrosa Rebouças Filho et al. proposed a different model based on structural co-occurrence matrix (SCM) to classify the skin lesions from dermoscopy images. They applied their method to the ISIC 2016, 2017 and PH2 datasets and then obtained very good results in the classification of skin lesions [8]. Jose Luis Garcia-Arroyo et al. proposed a segmentation method for skin lesions in the classification of melanoma disease from dermoscopy images. In their method, this approach has been based on the fuzzy classification of pixels and subsequent histogram thresholding [9]. In the other work [10], Serkan Sete et al. proposed a Gabor wavelet-based deep convolutional neural network for the classification of malignant melanoma and seborrheic keratosis from the dermoscopy images. Saptarshi Chatterjee et al. used a hybrid method for the classification of skin type lesions. In their method, for the feature extraction from the images, they used the gray level co-occurrence matrix (GLCM) and a fractal-based regional texture analysis (FRTA) algorithm. Then, to select important features from all feature sets, recursive feature elimination (RFE) has been used. To classify the skin lesions, the support vector machine with radial basis function (RBF) has been used in this paper [11]. Nazia Hameed et al. proposed a Multi-Class Multi-Level (MCML) classification algorithm for multi skin lesions [21]. In the other work of 
Pedro M.M. Pereira et al. [22], they used the Local Binary Pattern Clustering (LBPC) for segmentation of dermoscopy images. Then, they extracted some shape features from these images and classified them using the support vector machine and Feed Forward Network [22]. Javeria Amin et al. proposed a different method for localization and classification of skin cancer [23]. They applied the PCA to the images and then obtained new features to detect the skin cancer [23]. Ghasem Shakourian Ghalejoogh et al. proposed the Stacking Ensemble Method based on the Meta Learning algorithm for skin disease classification from the dermoscopy images. In this dataset, three classes including benign, dysplastic and melanoma have been used [24]. In the work of Muhammad Attique Khan et al. [25], they proposed a hybrid approach including faster region based convolutional neural network (RCNN), deep feature extraction, and feature selection by IcNR approach for skin lesion classification. Fengying Xie et al. proposed a new segmentation method called the Convolutional neural network and then applied it to skin images dataset [26]. Ahmed Refaat Hawas et al. proposed a histogram-based clustering estimation (HBCE) algorithm for skin lesion segmentation. And then, they combined it with neutrosophic c-means (NCM) for segmentation of dermoscopic images [27]. As for the work of Teck Yan Tan et al. [28], they proposed a hybrid Particle Swarm Optimization (HPSO) for the segmentation of skin lesions and then combined it with CNN algorithm for detection of skin lesions.

Apart from the literature works, we have classified the seven different skin diseases including Actinic keratoses and intraepithelial carcinoma (akiec), Basal cell carcinoma (bcc), Benign keratosis (bkl), Dermatofibroma (df), Melanoma (mel), Melanocytic type (nv) and Vascular lesions (vasc) using two different CNN models. In general, in the conducted works in the literature, two different skin diseases have been classified using the dermoscopy images. In this paper, we have focused on the multi-class classification problem named skin disease detection using two different approaches.

The novelty and the contributions of this work are:

$>$ Two different models have been proposed for seven different skin diseases using deep learning methods. In the literature, generally, two different skin diseases have been classified.

$>$ We have firstly combined the convolutional neural network (CNN) and one-versus-all (OVA) to classify the multi-class skin disease dataset with high performance.

$>$ We have not used any pre-processing method in the dermoscopy images.

The rest of this study is organized as follows: the details of the dataset and methods are given in Section 2. The experimental results and discussion are presented in Section 3. Lastly, concluding remarks are given in Section 4.

\section{Material and Method}

\subsection{Dataset}

In this study, we have used the HAM10000 dataset for dermoscopy images [12]. It contains pigmented lesions in different populations. This data set consists of 10015 images in total. The image sizes are scaled to $150 \times 200$ pixels to reduce network input and reduce the number of network parameters. The diagnostic classes in this dataset are given as follows [12]. 

a) Actinic keratoses and intraepithelial carcinoma - akiec
b) Basal cell carcinoma - bcc
c) Benign keratosis - bkl
d) Dermatofibroma - df
e) Melanoma -mel
f) Melanocytic type - nv
g) Vascular lesions - vasc

Figure 1 shows the akiec example taken from [12]. Figure 2 denotes the bcc example taken from [12]. Figure 3 gives the bkl example taken from [12]. As for figure 4, the df example is shown. Figure 5 shows the mel example taken from [12]. Figure 6 explains the nv example taken from [12]. The vasc example is given in Figure 7.
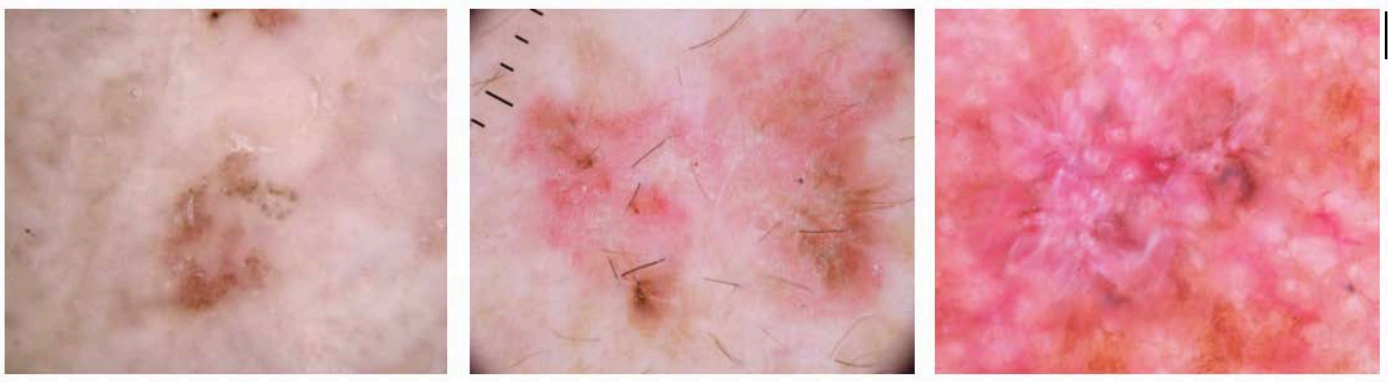

Figure 1. The dataset example taken from: akiec [12]
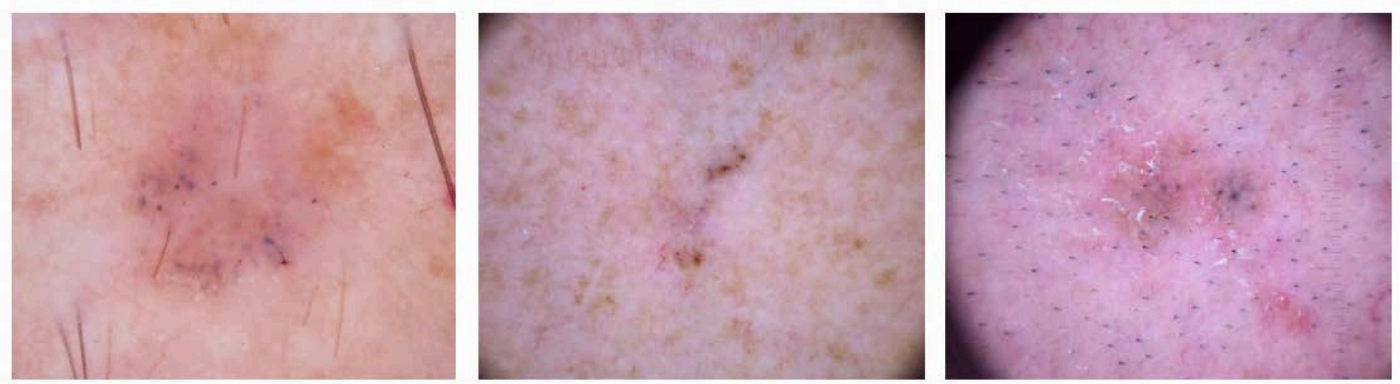

Figure 2. The dataset example taken from: bcc [12]
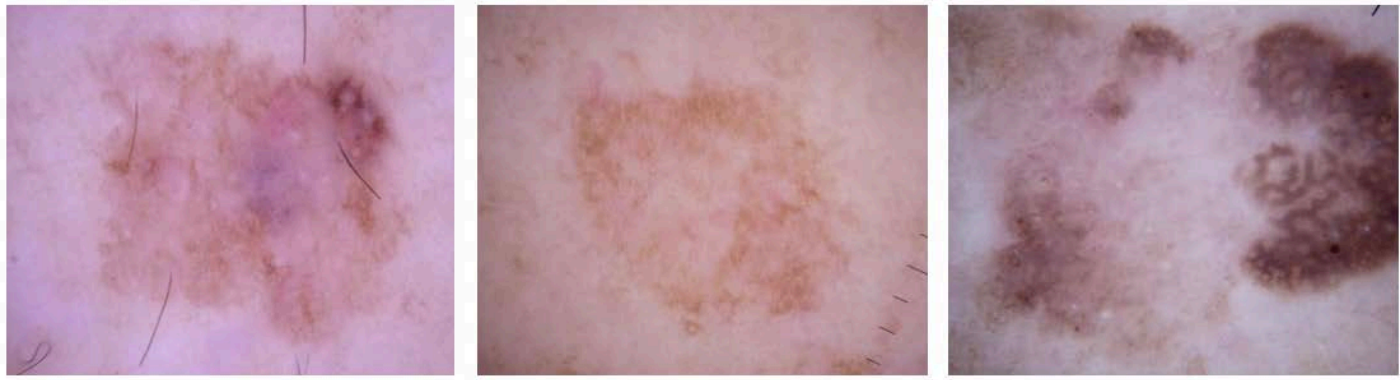

Figure 3. The dataset example taken from: bkl [12] 

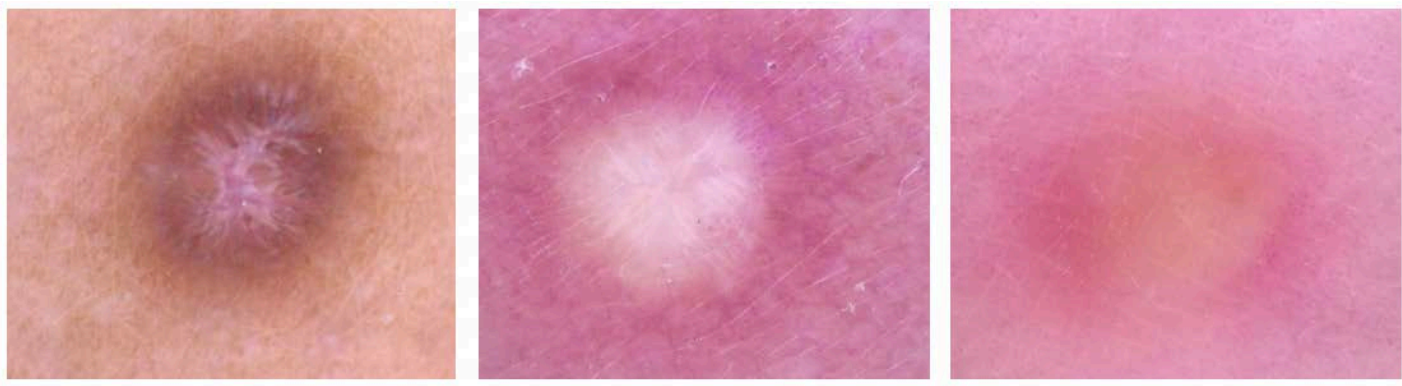

Figure 4. The dataset example taken from: df [12]
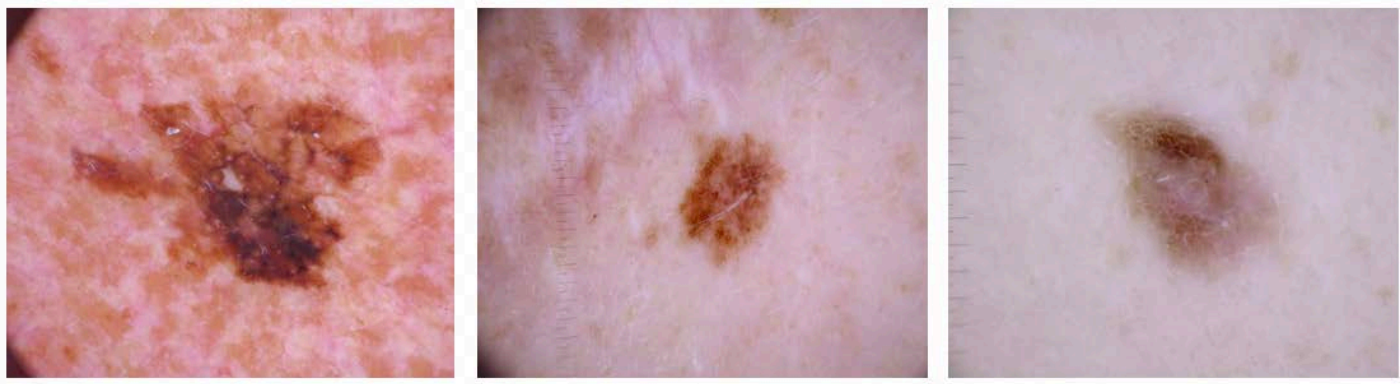

Figure 5. The dataset example taken from: mel [12]
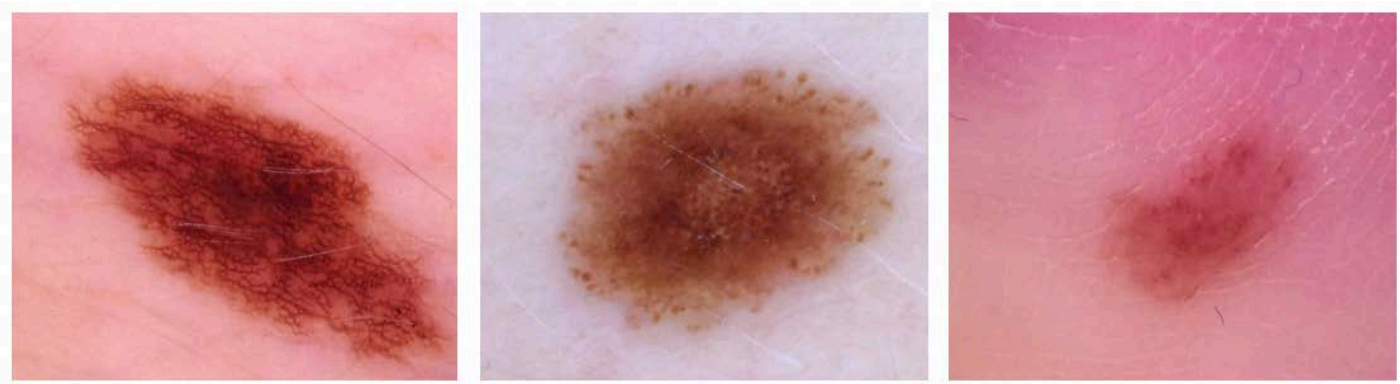

Figure 6. The dataset example taken from: nv [12]
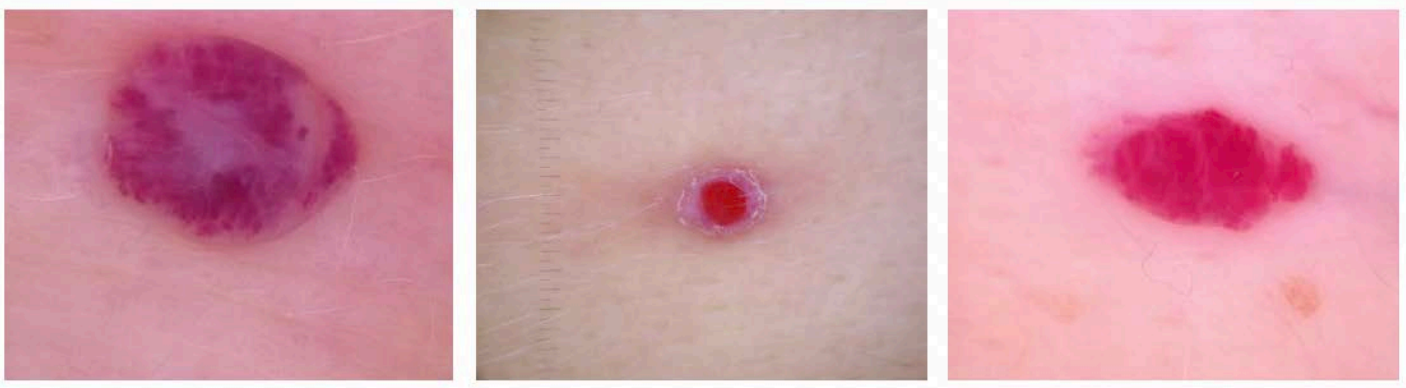

Figure 7. The dataset example taken from: vasc [12]

Information about which class belongs to each image in the dataset is also provided with the dataset. The sets to be used in the portions of the dataset were obtained from the entire data set to $75 \%, 5 \%$, and $20 \%$, respectively, training, verification and testing. Data indicating how much data each set contains are listed in Table 1. 
Table 1. Data indicating how many data each set contains in the dataset [12]

\begin{tabular}{|l|c|c|c|}
\hline \multicolumn{1}{|c|}{ Diagnosis Class in the dataset } & Train & Validity & Test \\
\hline Akiec & 243 & 17 & 67 \\
\hline Bcc & 386 & 25 & 103 \\
\hline Bkl & 814 & 59 & 225 \\
\hline Df & 79 & 9 & 27 \\
\hline Mel & 844 & 52 & 217 \\
\hline Nv & 5034 & 328 & 1343 \\
\hline Vasc & 111 & 10 & 21 \\
\hline Total & 7511 & 500 & 2003 \\
\hline
\end{tabular}

As can be seen in the table, most of the data in the dataset belong to the sixth grade, and there is no equal distribution between classes. During this training phase, it will lead to more learning of the 6th grade and the network to learn this information and to learn this class. In order to overcome this problem, the number of images in the training set has been increased by using the data duplication method. The resulting training set contains 10015 images.

\subsection{The Proposed Method}

To classify the skin diseases automatically, two different methods have been proposed: i) Alone CNN model, and ii) the combination of CNN and oneversus- all (OVA). In the proposed method, we have not used any pre-processing method to classify them. The raw dermatology images taken from the dataset have been given to the input of Convolutional Neural Network and then trained and tested by these images. In the second proposed method, seven different models having two-classes have been composed and then combined with the one-versus-all approach. As the pre-processing stage, we have just used the normalization method.

In this paper, the first method has been proposed to classify the images in the dataset as multi-class using the convolutional neural network (CNN). In this method, the input image has been normalized in the range of $0-1$, then applied as an input to the designed CNN network. At the output of the CNN, information about which class the image belongs to is obtained. The flow diagram of the first method is given in Figure 8. 


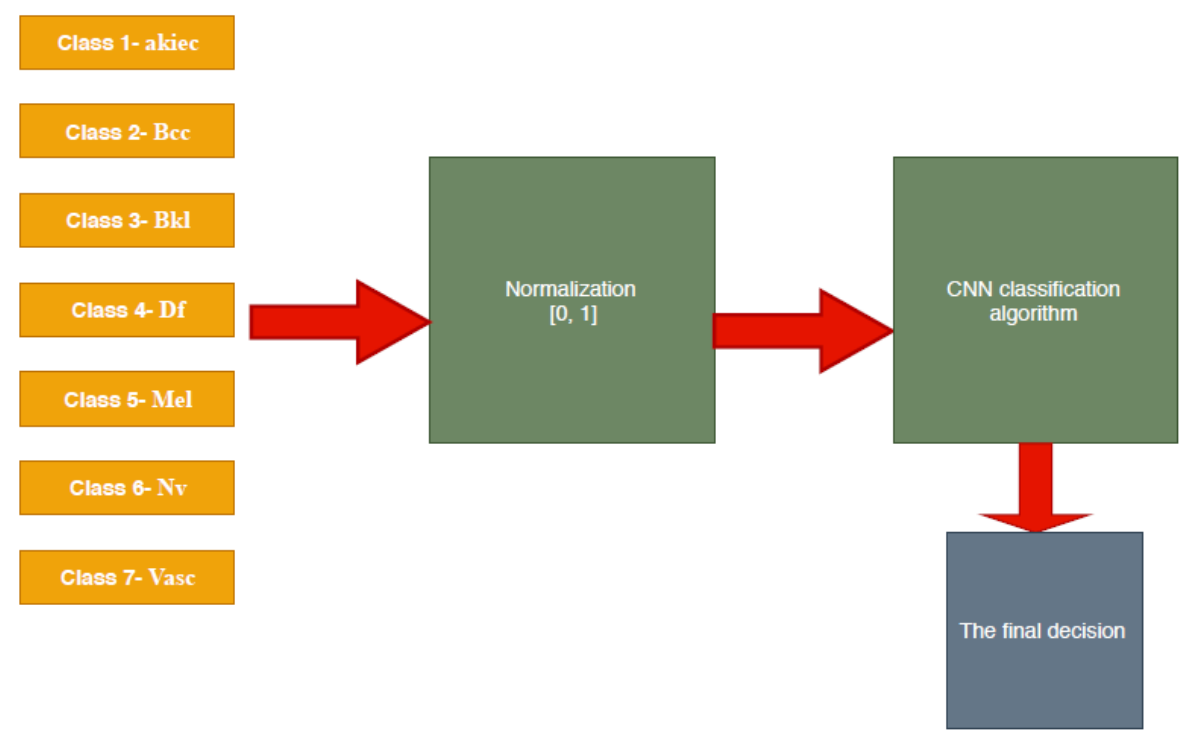

Figure 8. The flow diagram of the first method (alone CNN)

In the second method, CNN has been combined with one versus all approach to improving the classification performance. In this method, we have classified all the classes as the pairwise: Class 1 - other (a combination of other classes in the dataset), C2- other (a combination of other classes in the dataset), C3- other (a combination of other classes in the dataset), and so on. Then, we have taken an average of all the pairwise results. Thanks to the proposed second method, the classification performance has been increased from $77 \%$ accuracy to $92.90 \%$ accuracy. The flow diagram of the second method is given in Figure 9.

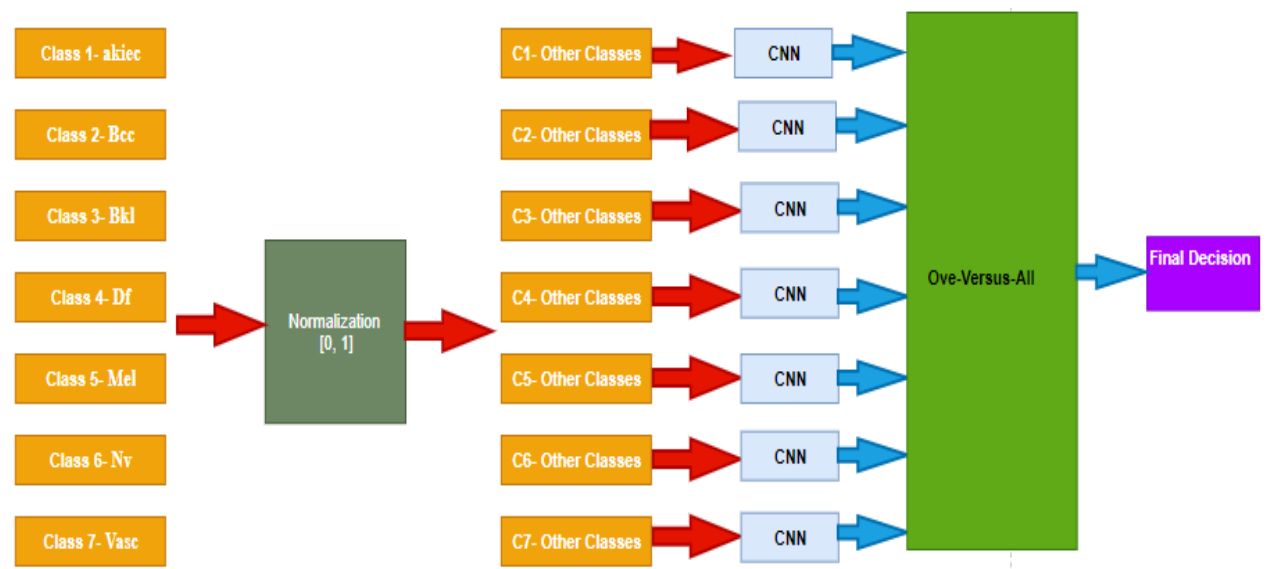

Figure 9. The flow diagram of the second method (the combination of CNN and OVA)

With the introduction of deep convolutional neural networks in image processing applications, high-performance results have begun to be achieved in many image processing problems [9]. These networks mainly contain two different structures, namely convolution and neural network. While convolutional structures allow imitation of the capabilities of image processing techniques by networks, neural networks are used to make sense of this data obtained from images. These structures are mentioned in the next subsections. 
The first network structure consists of four different evolutionary layers and fully connected layers added to the output of these layers. This network structure includes 1,243,463 parameters in total, and the visualized structure of the network is given in Figure 10.

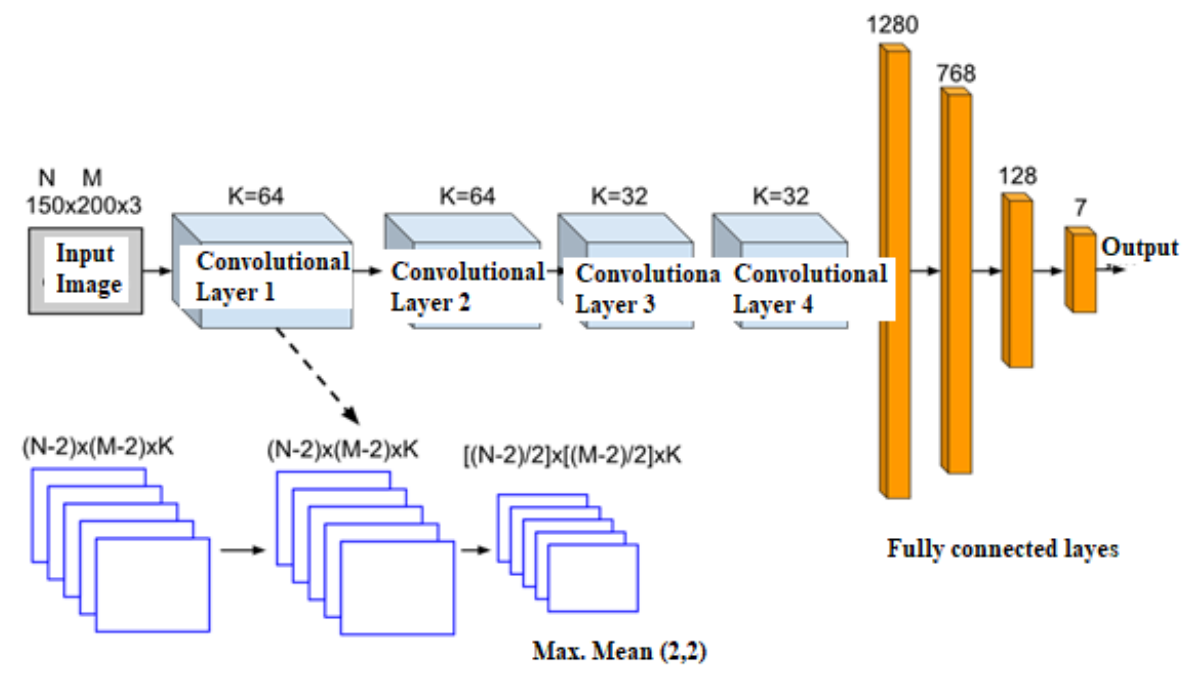

Figure 10. The proposed network structure model-1 for our problem

Using the network structure given in the figure, training was carried out for each class by using a data set consisting of all other classes against one class. For each class, binary (coded as 10-true, 01-false in the study) classification is made at the output of the network. In this way, seven different pieces of training were carried out for seven classes. It can be understood whether an input image of the relevant class is given at the exit of each network. This network structure contains 153090 parameters in total, and the visualized structure of the network is given in Figure 11.

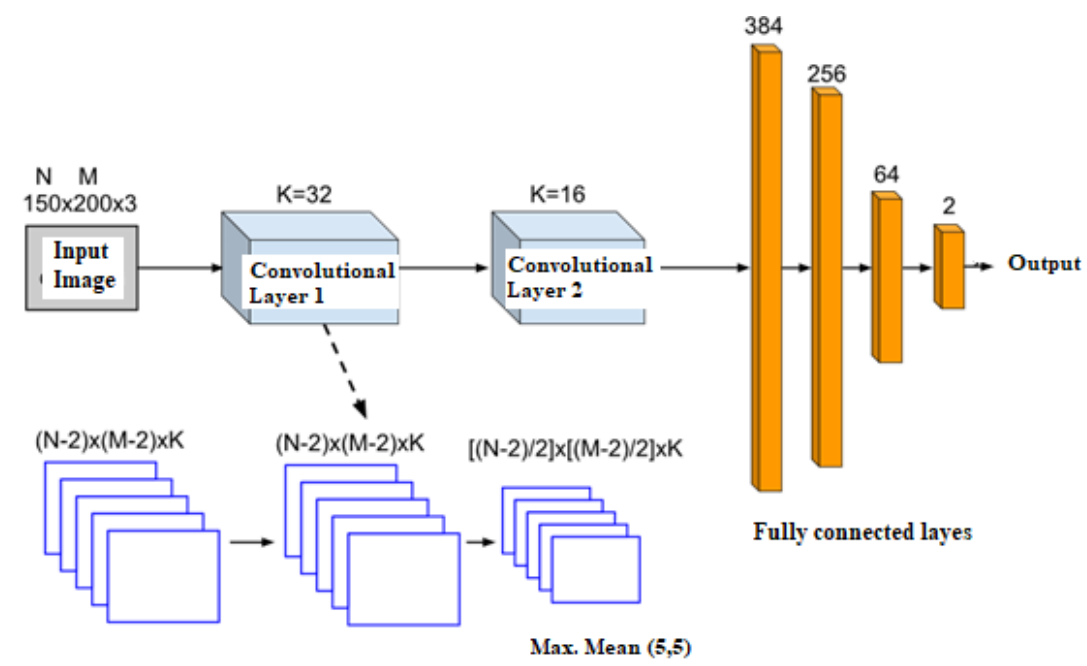

Figure 11. The proposed network structure model-2 for our problem 


\subsubsection{Data Augmentation}

Neural networks need to be well trained to achieve successful results from processing large amounts of data [15]. The number of images used for education may have insufficient or unbalanced distribution. In order to increase the number of insufficient data used in education, replication is performed in the data set. The data replication process is used to create additional data for the training set. It is realized by different approaches such as rotating, scaling, translation, adding noise on the original image, or combining these approaches in combination [15, 29, 30]. The training set with the data replication process has the number of images in Table 2. The specified training set was used for the training. A cycle is completed using the entire training dataset in groups of 32 images for training. A total of 15 cycles were made for training. At the end of each cycle, verification results are obtained by using the verification set. This value indicates how the network has achieved the data it has not seen before. The education coefficient was chosen as 0.0001 .

Table 2. The distribution of data after the data augmentation

\begin{tabular}{|l|c|}
\hline \multicolumn{1}{|c|}{ Diagnosis Class in the dataset } & Number of data after the augmentation \\
\hline Akiec & 4800 \\
\hline Bcc & 5018 \\
\hline Bkl & 4884 \\
\hline Df & 4977 \\
\hline Mel & 4220 \\
\hline Nv & 5034 \\
\hline Vasc & 4995 \\
\hline Total & 33998 \\
\hline
\end{tabular}

\section{The Experimental Results and Discussion}

In this study, the confusion matrix is used to calculate several metrics. This matrix forms four indices which are true positive (TP), false positive (FP), false negative (FN) and true negative (TN). TP and TN match the number of correctly predicted hypoxic and normal samples whereas the FP and FN match the number of incorrectly predicted hypoxic and normal samples, respectively. These metrics, their mathematical forms, and short descriptions are given in Table 3.

Table 3. The used performance metrics with their descriptions in this work

\begin{tabular}{|l|c|l|}
\hline \multicolumn{1}{|c|}{ Metrics } & Equations & \multicolumn{1}{c|}{ Descriptions } \\
\hline Accuracy & $\frac{T P+T N}{T P+F P+F N+T N}$ & The overall accuracy of the model. \\
\hline Sensitivity (Recall) & $\frac{T P}{T P+F N}$ & The capacity of the model on the prediction of the hypoxic fetus. \\
\hline Specificity & $\frac{T N}{T N+F P}$ & The capacity of the model on the prediction of the normal fetus. \\
\hline
\end{tabular}




\begin{tabular}{|l|l|l|}
\hline Precision & $\frac{T P}{T P+F P}$ & The correctly predicted hypoxic fetus. \\
\hline F-Score & $\frac{2 * P * R}{P+R}$ & The harmonic mean between precision and recall. \\
\hline
\end{tabular}

The meanings of the classification results obtained from the trained networks and their outlets are shown in the figure. The education of a dataset containing two classes is easier than a multi-class dataset. There is no need for the wide network structure used previously. You can see the test achievements obtained from the network structures trained for each class in Table 4 and Table 5 . The simulations were conducted in the TensorFlow Deep learning. The computation cost for each model is approximately 4 hours.

Table 4. Performance indicators of the first network for each class in the detection of skin diseases using CNN model-1

\begin{tabular}{|l|c|c|c|}
\multicolumn{1}{|c|}{$\begin{array}{c}\text { Diagnosis Class } \\
\text { in the dataset }\end{array}$} & Precision & Recall & F score \\
\hline Akiec & 0.58 & 0.21 & 0.31 \\
\hline Bcc & 0.64 & 0.59 & 0.62 \\
\hline Bkl & 0.48 & 0.61 & 0.54 \\
\hline Df & 0.39 & 0.41 & 0.40 \\
\hline Mel & 0.51 & 0.42 & 0.46 \\
\hline Nv & 0.89 & 0.91 & 0.90 \\
\hline Vasc & 1.00 & 0.48 & 0.65 \\
\hline
\end{tabular}

Table 5. Performance indicators of the second network for each class in the detection of skin diseases using CNN model-2

\begin{tabular}{|l|c|}
\hline \multicolumn{1}{|c|}{$\begin{array}{c}\text { Diagnosis Class } \\
\text { in the dataset }\end{array}$} & Precision \\
\hline Akiec & $96.9 \%$ \\
\hline Bcc & $95 \%$ \\
\hline Bkl & $89.7 \%$ \\
\hline Df & $98.9 \%$ \\
\hline Mel & $87.70 \%$ \\
\hline Nv & $82.90 \%$ \\
\hline Vasc & $99.20 \%$ \\
\hline Average & $92.90 \%$ \\
\hline
\end{tabular}

Figure 12 shows the performance graph of the proposed first model (alone CNN) in the classification of skin diseases having seven classes. As for the other proposed method called the combination of CNN and OVA, the obtained performance graphs are given in Figures 13 through 19. for each class in the skin disease dataset. Figure 20 shows the error diagram of each class in the classification of skin diseases using 
the CNN method.

The performance graph of the proposed first model (alone $\mathrm{CNN}$ ) in the classification of skin diseases

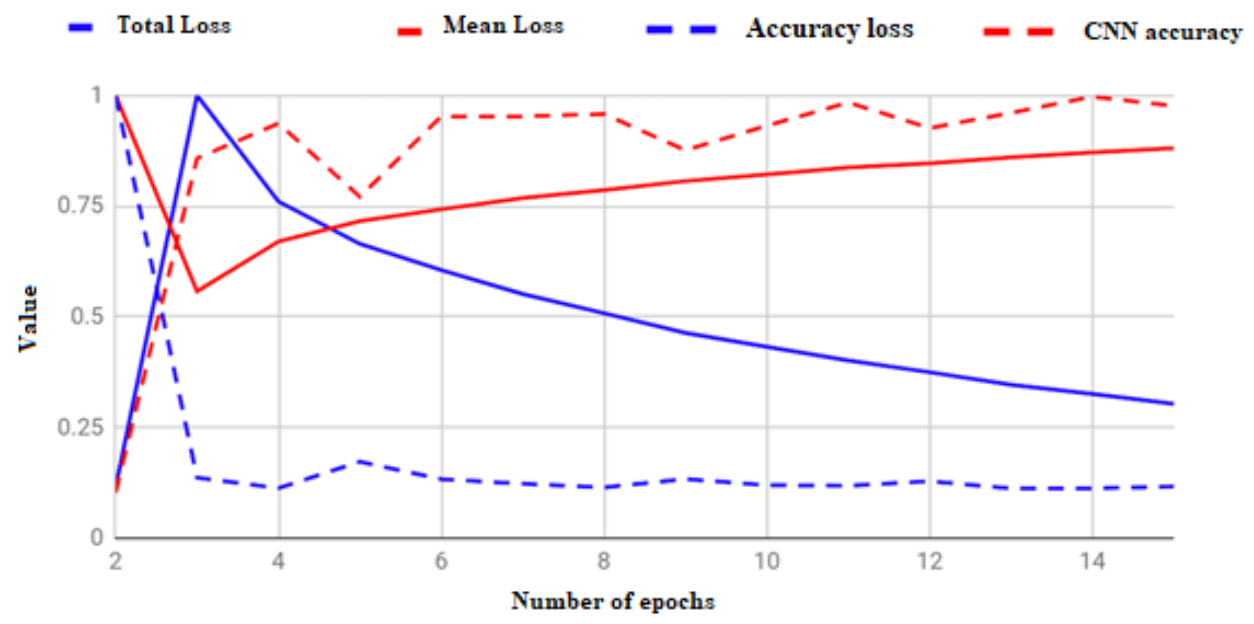

Figure 12. The performance graph of the proposed first model (alone CNN) in the classification of skin diseases having seven classes

The performance graph of the second proposed method (CNN-OVA) in the classification of first class and other classes of dataset

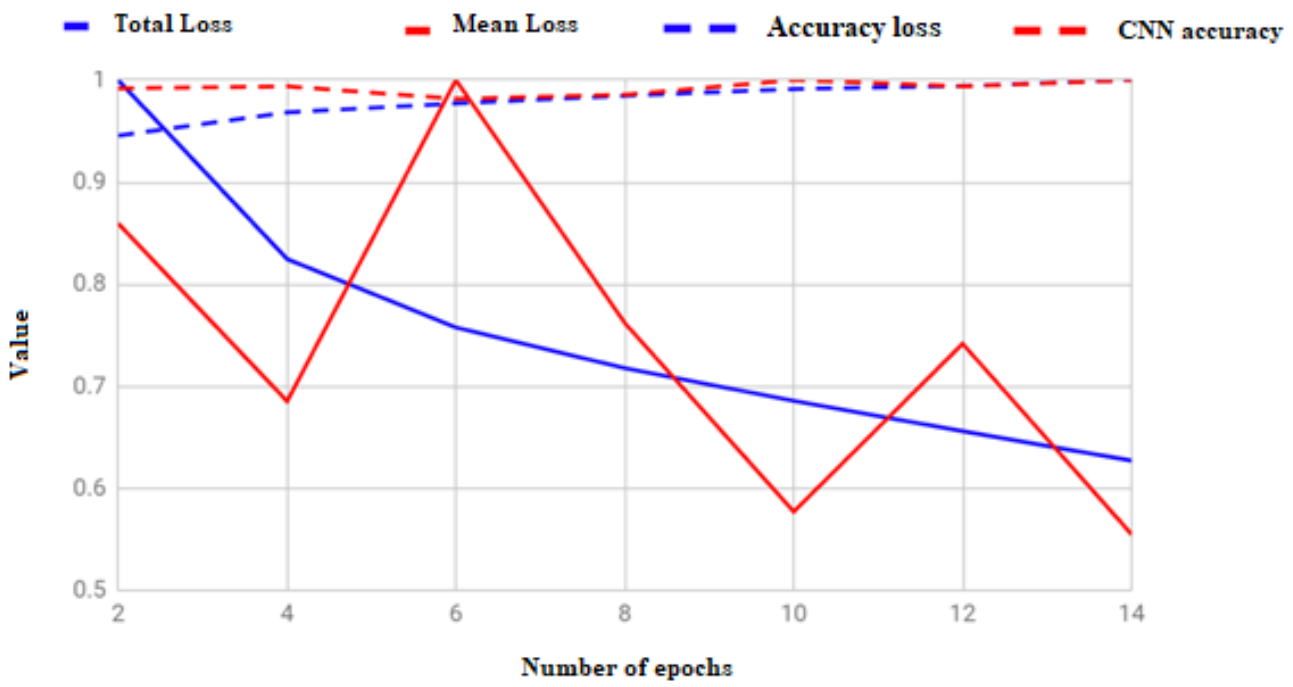

Figure 13. The performance graph of the proposed second model (the combination of CNN and OVA) in the classification of first class (Akiec) from skin diseases having seven classes 


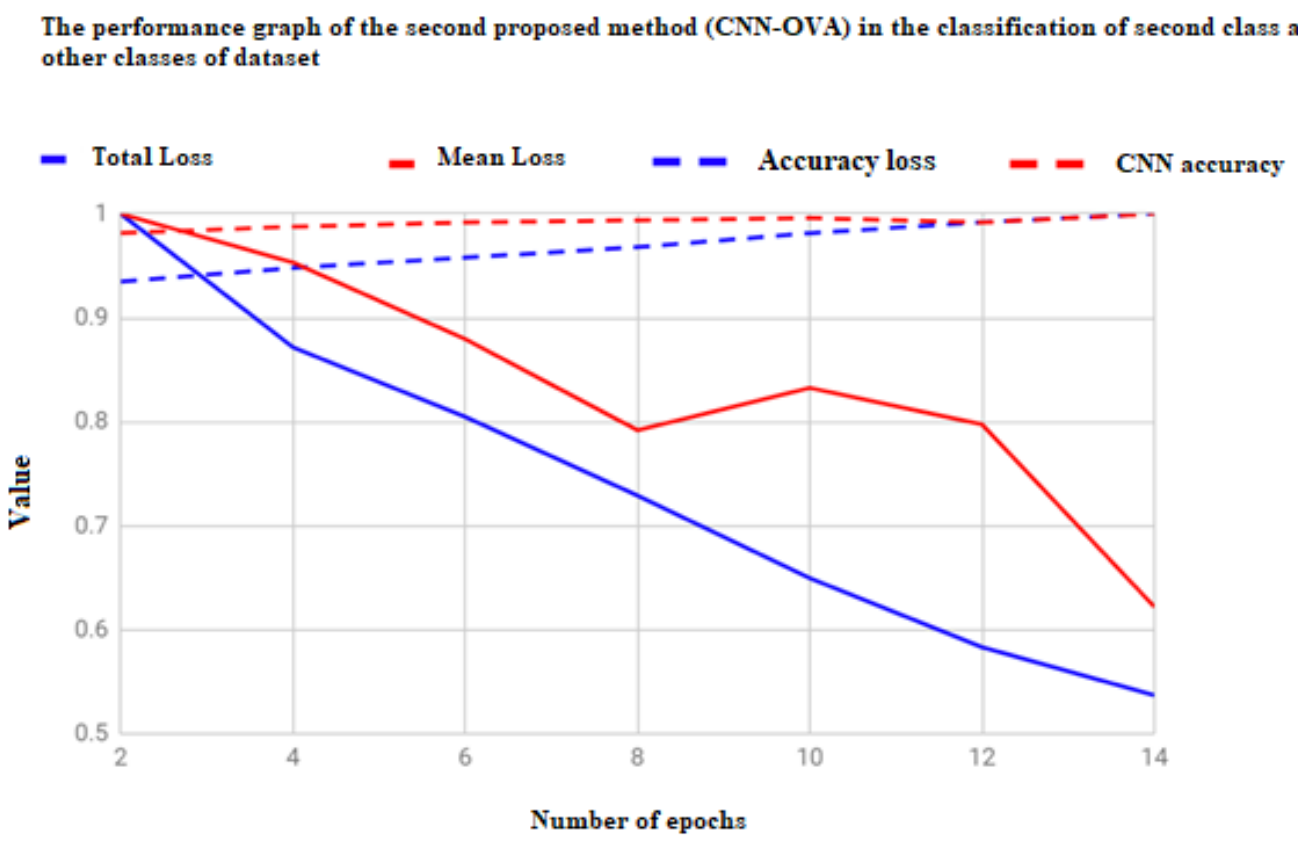

Figure 14. The performance graph of the proposed second model (the combination of CNN and OVA) in the classification of second class (Bcc) from skin diseases having seven classes

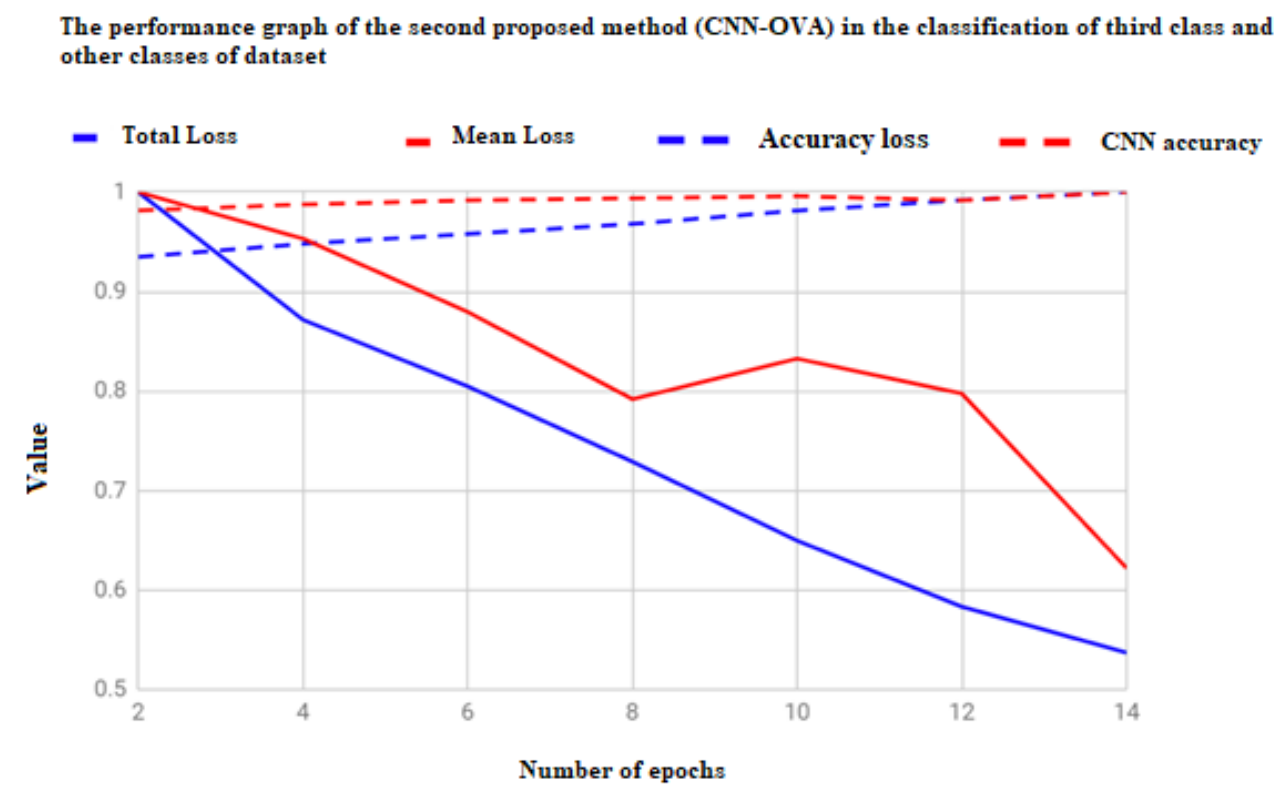

Figure 15. The performance graph of the proposed second model (the combination of CNN and OVA) in the classification of third class (Bkl) from skin diseases having seven classes 


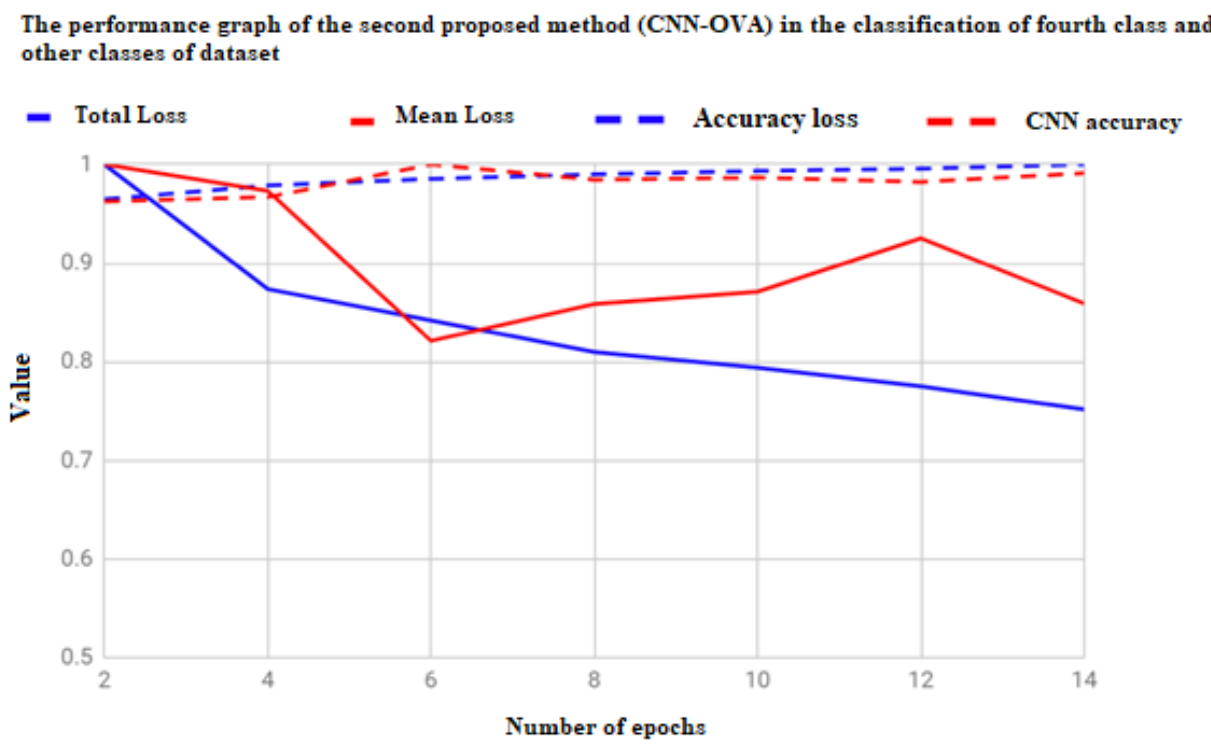

Figure 16. The performance graph of the proposed second model (the combination of CNN and OVA) in the classification of fourth class (Df) from skin diseases having seven classes

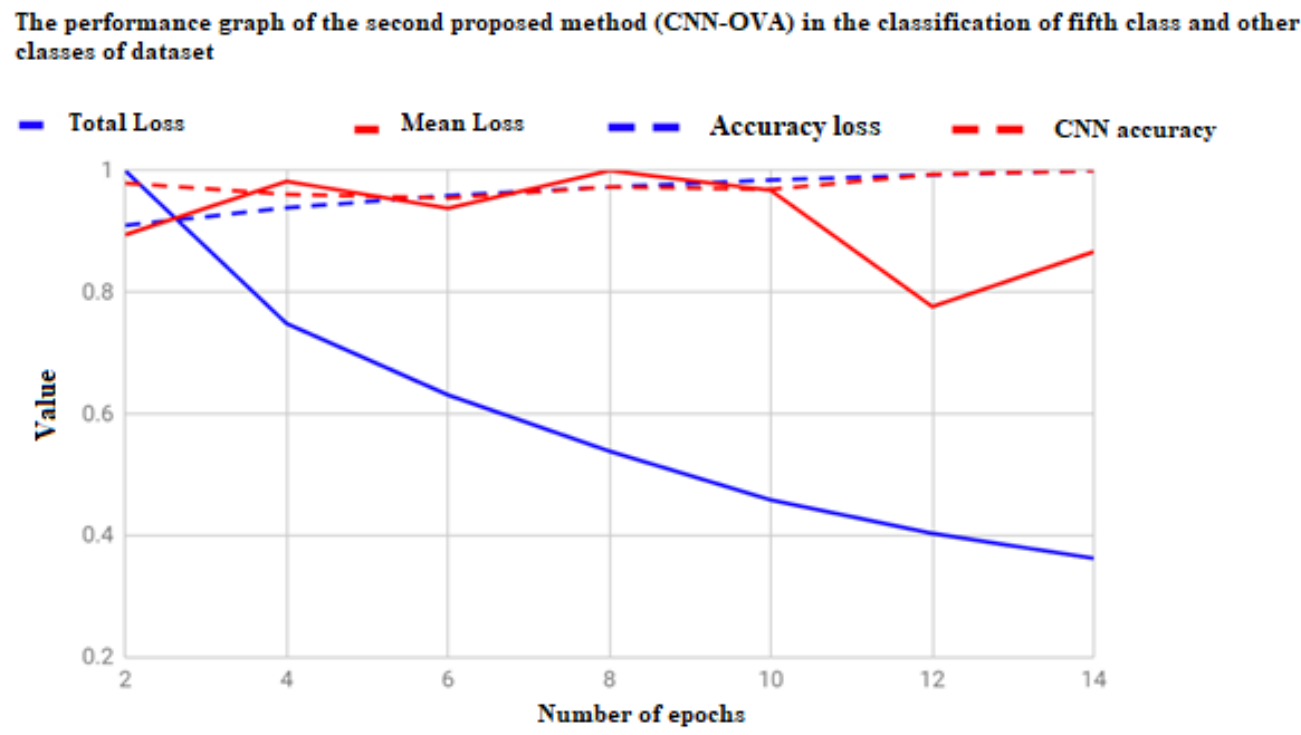

Figure 17. The performance graph of the proposed second model (the combination of CNN and OVA) in the classification of fifth class (Mel) from skin diseases having seven classes 


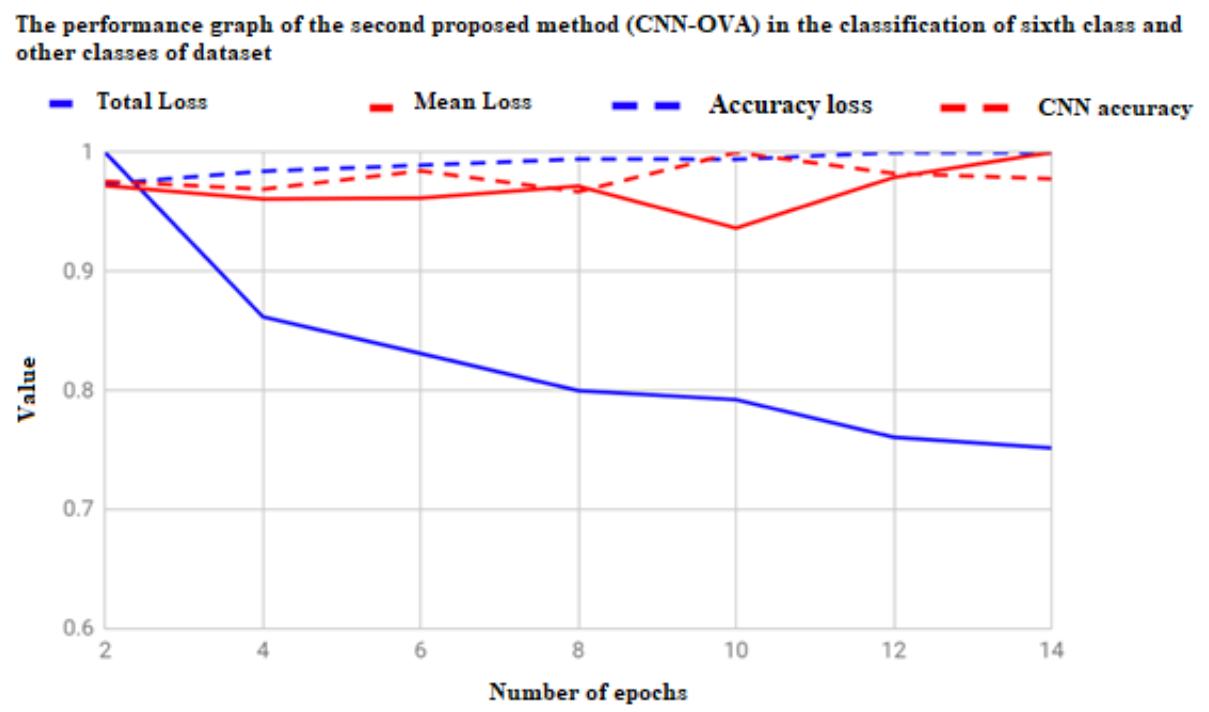

Figure 18. The performance graph of the proposed second model (the combination of CNN and OVA) in the classification of sixth class ( $\mathrm{Nv}$ ) from skin diseases having seven classes

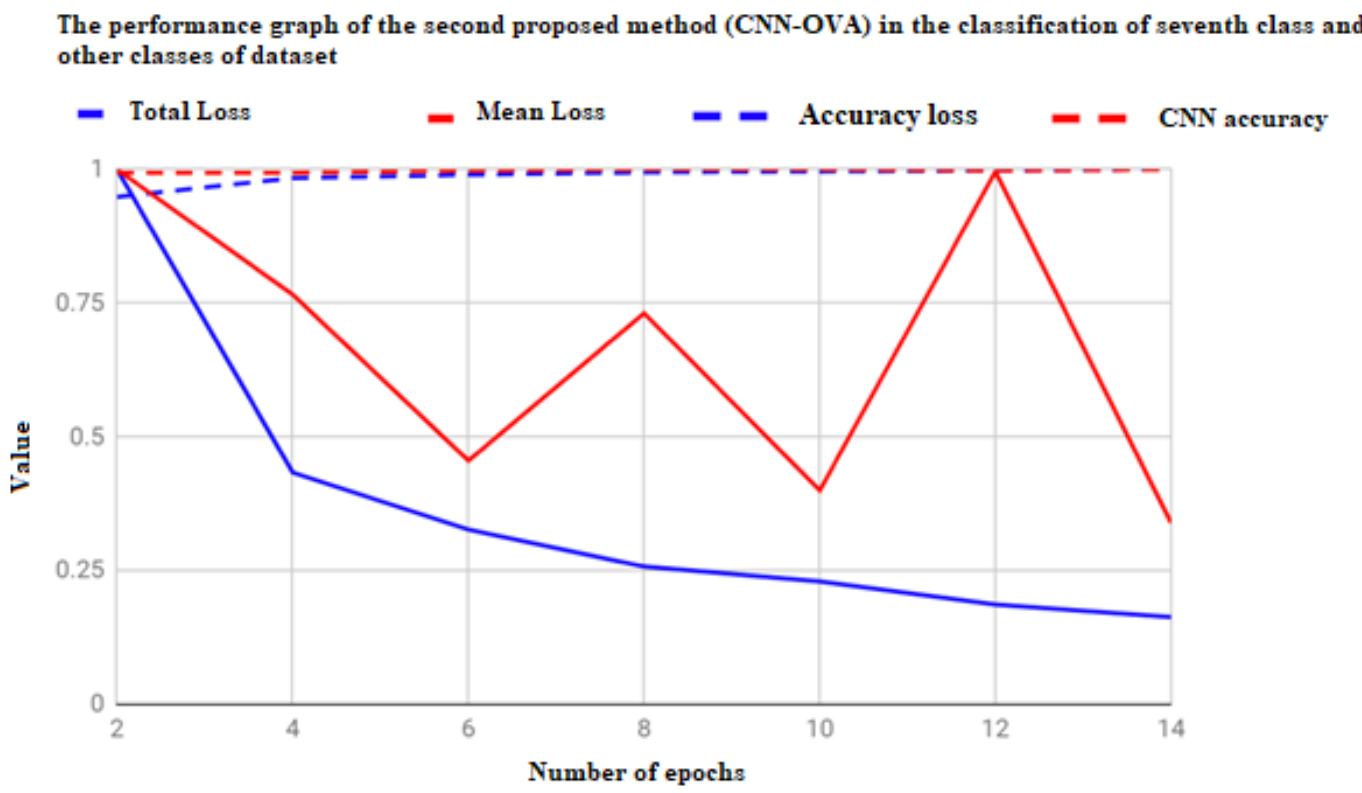

Figure 19. The performance graph of the proposed second model (the combination of CNN and OVA) in the classification of seventh class (Vasc) from skin diseases having seven classes 


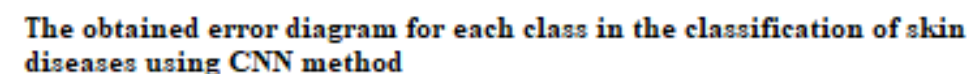

diseases using $\mathrm{CNN}$ method

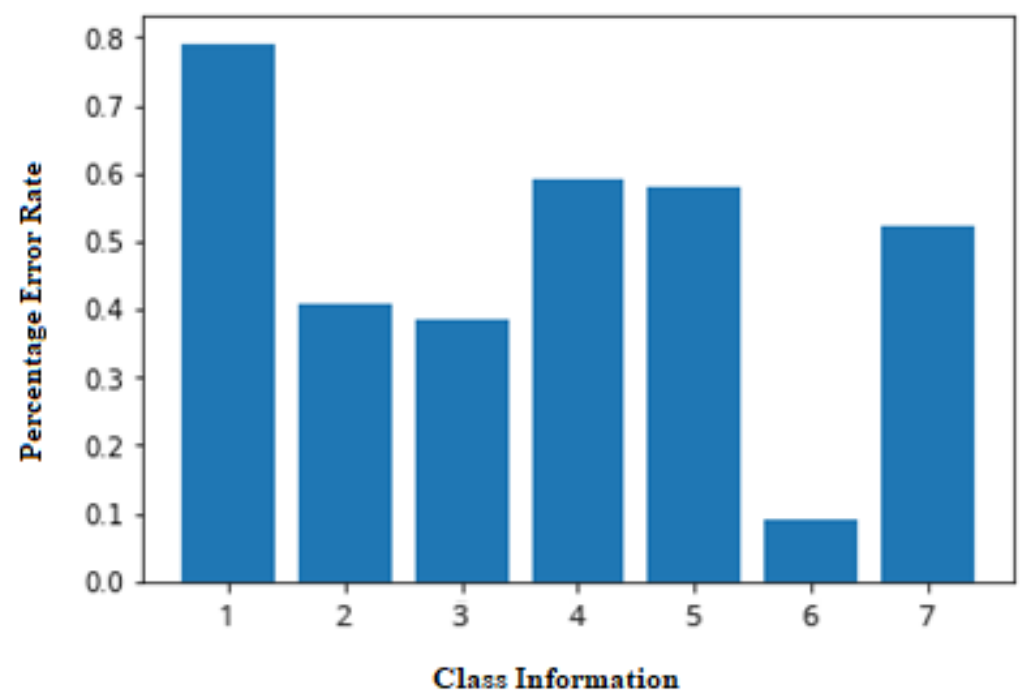

Figure 20. The error diagram of each class in the classification of skin diseases using CNN method

When the two proposed models in the study are compared; the first network structure has achieved $77 \%$ success as a result of separating and classifying seven classes in total. In the second model, when each class is evaluated as one, respectively, higher success rates are observed. By collecting the success rates of the mentioned seven classes, the average of the proposed second network was $92.9 \%$. When the second network, which is among the proposed networks, is evaluated by considering similar studies in the literature, it has achieved success in a value that will contribute to the literature. The values related to the comparison are shown in Table 6. As can be seen from the obtained results, the best method is our method called the combination of CNN and one versus all (OVA) in the classification of skin diseases from the dermoscopy images.

Table 6. Performance comparison of the second model (the combination of CNN and one versus all) with the literature

\begin{tabular}{|l|c|}
\hline \multicolumn{1}{|c|}{ The relevant work } & $\begin{array}{c}\text { The obtained classification } \\
\text { accuracy (\%) }\end{array}$ \\
\hline Aleksey Nozdryn-Plotnick et al. [16] & 88.50 \\
\hline Nils Gessert et al. [17] & 85.60 \\
\hline Iaxin Zhuang et al. [18] & 84.50 \\
\hline Mohammed K. Amro et al. [19] & 81.20 \\
\hline Philipp Tschandl et al. [14] & 80.20 \\
\hline Yeong Chan Lee et al. [20] & 78.50 \\
\hline Aminur Rab Ratul et al. [13] & 87.42 \\
\hline Aminur Rab Ratul et al. [13] & 85.02 \\
\hline
\end{tabular}




\begin{tabular}{|l|c|}
\hline Aminur Rab Ratul et al. [13] & 88.22 \\
\hline Aminur Rab Ratul et al. [13] & 89.81 \\
\hline Our study (2020) & 92.90 \\
\hline
\end{tabular}

\section{Conclusions}

In the paper, we have proposed two different approaches based on CNN and one-versus-all (OVA) for the classification of skin diseases from dermoscopy images. Apart from the literature, the skin diseases dataset having seven classes has been classified by the combination of CNN and OVA with a high classification performance. The simulations of this work have been done in Tensor Flow deep learning. Without using any filtering and any feature extraction, we have obtained very promising results in the detection of skin lesions. The advantage of OVA is that classification has been made based on true and false for each class. Therefore, it caused the complexity to decrease. Thus, the accuracy rate values in multiple classifications increased when each class was evaluated separately. While alone CNN obtained 77\% classification accuracy in the detection of skin disease with 7 classes, the combination of CNN and one-versus-all approach achieved 92.90\% accuracy. The proposed method, called the combination of CNN and OVA could be used in many medical imaging classification problems.

\section{Conflicts of Interest}

There is no conflict of interest.

\section{References}

[1] Halk Sağlı̆̆ı Genel Müdürlüğü-https://hsgm.saglik.gov.tr, (last accessed: January, 2020).

[2] Oktay Yıldı, Melanoma detection from dermoscopy images with deep learning methods: Acomprehensive study, Journal of the Faculty of Engineering and Architecture of Gazi University 34:4 (2019) 2241-2260.

[3] M. Emre Celebi, Hassan A. Kingravi, Bakhtiyar Uddin, Hitoshi Iyatomi, Y. Alp Aslandogan, William V. Stoecker, Randy H. Moss, A methodological approach to the classification of dermoscopy images, Computerized Medical Imaging and Graphics, 31(6), 2007, 362-373.

[4] https://dermnetnz.org/topics/convolutional-neural-networks-in-dermatology/, (last accessed: January, 2020).

[5] Suhail M. Odeh, Abdel Karim Mohamed Baareh, A comparison of classification methods as diagnostic system: A case study on skin lesions, Computer Methods and Programs in Biomedicine, 137, 2016, 311-319.

[6] Fekrache Dalila, Ameur Zohra, Kasmi Reda, Cherifi Hocine, Segmentation and classification of melanoma and benign skin lesions, Optik, 140, 2017, 749-761.

[7] Wiem Abbes, Dorra Sellami, Automatic Skin Lesions Classification Using Ontology-Based Semantic Analysis of Optical Standard Images,Procedia Computer Science, 112, 2017, 2096-2105.

[8] Pedro Pedrosa Rebouças Filho, Solon Alves Peixoto, Raul Victor Medeiros da Nóbrega, D. Jude Hemanth, Aldisio Gonçalves Medeiros, Arun Kumar Sangaiah, Victor Hugo C. de Albuquerque, Automatic histologically-closer 
classification of skin lesions, Computerized Medical Imaging and Graphics, 68, 2018, 40-54.

[9] Jose Luis Garcia-Arroyo, Begonya Garcia-Zapirain, Segmentation of skin lesions in dermoscopy images using fuzzy classification of pixels and histogram thresholding, Computer Methods and Programs in Biomedicine, 168, 2019, 11-19.

[10] Sertan Serte, Hasan Demirel, Gabor wavelet-based deep learning for skin lesion classification, Computers in Biology and Medicine, 113, 2019, 103423.

[11] Saptarshi Chatterjee, Debangshu Dey, Sugata Munshi, Integration of morphological preprocessing and fractal based feature extraction with recursive feature elimination for skin lesion types classification, Computer Methods and Programs in Biomedicine, 178, 2019, 201-218.

[12] Tschandl, P., Rosendahl, C. \& Kittler, H. The HAM10000 dataset, a large collection of multi-source dermatoscopic images of common pigmented skin lesions. Sci Data 5, 180161 (2018). https://doi.org/10.1038/sdata.2018.161.

[13] Skin Lesions Classification Using Deep Learning Based on Dilated Convolution Aminur Rab Ratul, M. Hamed Mozaffari, Won-Sook Lee, Enea Parimbelli bioRxiv 860700; doi: https://doi.org/10.1101/860700.

[14] Philipp Tschandl, Christoph Sinz, Harald Kittler, Domain-specific classification-pretrained fully convolutional network encoders for skin lesion segmentation, Computers in Biology and Medicine, 104, 2019, 111-116.

[15] Goodfellow, I., Bengio, Y. and Courville, A. "Deep Learning, The MIT Press” (2016).

[16] Aleksey Nozdryn-Plotnicki, Jordan Yap, and William Yolland "Ensembling Convolutional Neural Networks for Skin Cancer Classification“ (2018), https://arxiv.org/abs/1808.05071.

[17] Nils Gessert, Thilo Sentkerac, Frederic Madestaac, Rudiger Schmitz " ad, Helge Kniepag, Ivo Baltruschataef, Rene Werner ' ac and Alexander Schlaeferb “ Skin Lesion Diagnosis using Ensembles, Unscaled Multi-Crop Evaluation and Loss Weighting” (2018).

[18] Jiaxin Zhuang, Weipeng Li, Siyamalan Manivannan, Roy Wang, JianGuo Zhang, Jihan Liu, Jiahui Pan, Gongfa Jiang, Ziyu Yin "Skin Lesion Analysis Towards Melanoma Detection Using Deep Neural Network Ensemble ” (2018)

[19] Mohammed K. Amro, Baljit Singh, and Avez Rizvi "Skin Lesion Classification and Segmentation for Imbalanced Classes using Deep Learning " (2018)

[20] Yeong Chan Lee, Sang-Hyuk Jung, and Hong-Hee Won “ WonDerM: Skin Lesion Classification with Fine-tuned Neural Networks ” (2018).

[21] Nazia Hameed, Antesar M. Shabut, Miltu K. Ghosh, M.A. Hossain, Multi-class multi-level classification algorithm for skin lesions classification using machine learning techniques, Expert Systems with Applications, 141, 2020, 112961.

[22] Pedro M.M. Pereira, Rui Fonseca-Pinto, Rui Pedro Paiva, Pedro A.A. Assuncao, Luis M.N. Tavora, Lucas A. Thomaz, Sergio M.M. Faria, Skin lesion classification enhancement using border-line features - The melanoma vs nevus problem, Biomedical Signal Processing and Control, 57, 2020, 101765.

[23] Javeria Amin, Abida Sharif, Nadia Gul, Muhammad Almas Anjum, 
Muhammad Wasif Nisar, Faisal Azam, Syed Ahmad Chan Bukhari, Integrated design of deep features fusion for localization and classification of skin cancer, Pattern Recognition Letters, 131, 2020, 63-70.

[24] Ghasem Shakourian Ghalejoogh, Hussain Montazery Kordy, Farideh Ebrahimi, A hierarchical structure based on Stacking approach for skin lesion classification, Expert Systems with Applications, 145, 2020, 113127.

[25] Muhammad Attique Khan, Muhammad Sharif, Tallha Akram, Syed Ahmad Chan Bukhari, Ramesh Sunder Nayak, Developed Newton-Raphson based deep features selection framework for skin lesion recognition, Pattern Recognition Letters, 129, 2020, 293-303.

[26] Fengying Xie, Jiawen Yang, Jie Liu, Zhiguo Jiang, Yushan Zheng, Yukun Wang, Skin lesion segmentation using high-resolution convolutional neural network, Computer Methods and Programs in Biomedicine, 186, 2020, 105241.

[27] Ahmed Refaat Hawas, Yanhui Guo, Chunlai Du, Kemal Polat, Amira S. Ashour, OCE-NGC: A neutrosophic graph cut algorithm using optimized clustering estimation algorithm for dermoscopic skin lesion segmentation, Applied Soft Computing, 86, 2020, 105931.

[28] Teck Yan Tan, Li Zhang, Chee Peng Lim, Adaptive melanoma diagnosis using evolving clustering, ensemble and deep neural networks,Knowledge-Based Systems, 187, 2020, 104807.

[29] M. Nasir, M. Attique Khan, M. Sharif, I.U. Lali, T. Saba, T. Iqbal, An improved strategy for skin lesion detection and classification using uniform segmentation and feature selection based approach, Microscopy Research and Technique, 81 (6) (2018), pp. 528-543, 10.1002/jemt.23009

[30] L. Zhang, G. Yang, X.J. Ye, Automatic skin lesion segmentation by coupling deep fully convolutional networks and shallow network with textons, Journal of Medical Imaging, 6 (2) (2019). 